\title{
HMGA2 is associated with epithelial- mesenchymal transition and can predict poor prognosis in nasopharyngeal carcinoma
}

\author{
You-You Xia',* \\ Li Yin ${ }^{2, *}$ \\ Hao Tian' \\ Wen-Jie Guo² \\ Ning Jiang ${ }^{2}$ \\ Xue-Song Jiang ${ }^{2}$ \\ Jing $\mathrm{Wu}^{\prime}$ \\ Meng Chen' \\ Jian-Zhong $\mathrm{Wu}^{3}$ \\ Xia $\mathrm{He}^{2}$ \\ 'The Fourth Clinical Medical College \\ of Nanjing Medical University, \\ ${ }^{2}$ Department of Radiation Oncology, \\ ${ }^{3}$ Research Center of Clinical \\ Oncology, The Affiliated Jiangsu \\ Cancer Hospital of Nanjing Medical \\ University, Nanjing, Jiangsu, People's \\ Republic of China \\ *These authors contributed equally \\ to this paper
}

\author{
This article was published in the following Dove Press journal: \\ OncoTargets and Therapy \\ 17 January 2015 \\ Number of times this article has been viewed
}

\begin{abstract}
Objective: High-mobility group protein 2 (HMGA2) and epithelial-mesenchymal transition (EMT)-associated proteins play key roles in cancer progression and metastasis. However, the clinical significance of HMGA2 and its relationship with EMT markers in nasopharyngeal carcinoma (NPC) is unclear. This study aimed to assess the clinicopathological significance and prognostic value of HMGA2, E-cadherin, and vimentin in NPC.
\end{abstract}

Methods: Using immunohistochemistry, HMGA2, E-cadherin, and vimentin expression levels were evaluated in NPC $(n=124)$ and non-tumoral inflammatory nasopharynx $(n=20)$ tissues. The association of HMGA2 and EMT markers with clinicopathological characteristics and relationships between the protein levels and overall survival were analyzed.

Results: Compared with non-tumorous tissues, HMGA2 and vimentin levels were markedly increased in NPC tissues, whereas decreased E-cadherin levels were observed $(P<0.001)$. Moreover, HMGA2 expression was positively correlated with vimentin levels $(r=0.431$, $P<0.001)$ and negatively correlated with E-cadherin amounts $(r=-0.413, P<0.001)$ in NPC tissues. The expression of all three proteins correlated significantly with tumor $\mathrm{N}$ stage, TNM stage, and 2-year metastasis. Furthermore, significant correlations were found for T stage, $\mathrm{N}$ stage, TNM stage, HMGA2, E-cadherin, and vimentin (all $P<0.013$ ) with poor prognosis (univariate analysis). However, multivariate analyses showed that only HMGA2 (hazard ratio [HR]: 2.683, 95\% confidence interval [CI]: $1.185-6.077, P=0.018$ ) and $\mathrm{N}$ stage (HR: 7.892, 95\% CI: 2.731-22.807, $P<0.001$ ) were independent predictors of poor prognosis.

Conclusion: These results demonstrated that HMGA2, an independent prognostic factor, may promote NPC progression and metastasis, and is significantly associated with EMT proteins. Therefore, HMGA2 may be considered a potential therapeutic target in NPC.

Keywords: EMT, NPC, high-mobility group protein 2

\section{Introduction}

Nasopharyngeal carcinoma (NPC) is a common malignancy with remarkably distinctive ethnic and geographic distributions: it is highly prevalent in Southern China and Southeast Asia. ${ }^{1}$ Among head and neck cancers, most NPC cases are lowly differentiated or undifferentiated squamous cell carcinomas with a high tendency to metastasize to regional lymph nodes. ${ }^{2,3}$ In addition, early metastasis to the neck is common, with about $74.5 \%$ of patients presenting with regional lymph node metastasis at the time of diagnosis. ${ }^{4}$ Though NPC is sensitive to radiotherapy and/or chemotherapy, treatment failure remains high due to the development of local recurrence, lymph nodes, and distant metastasis. ${ }^{5}$
Correspondence: Xia He

Department of Radiation Oncology,

The Affiliated Jiangsu Cancer Hospital of Nanjing Medical University, 42 Bai Zi Ting Road, Nanjing, Jiangsu, People's Republic of China

Email hexia2003@tom.com 
Epithelial-mesenchymal transition (EMT) is an important process in tumor invasion and metastasis. It is defined by the loss of epithelial morphology and acquisition of a mesenchymal phenotype..$^{6-8}$ In the process of EMT, tumor cells escape from the primary site and invade the surrounding stroma, then enter blood or lymphatic vessels to establish new proliferating colonies. A significant hallmark of EMT is downregulation of the epithelial protein E-cadherin and upregulation of motile mesenchymal proteins such as vimentin. ${ }^{9,10}$ Moreover, E-cadherin or vimentin expression has been associated with metastatic dissemination and overall survival (OS) in some solid tumor types, including soft tissue leiomyosarcoma, non-small cell lung cancer, unknown primary cancers, and NPC. ${ }^{11-14}$

High-mobility group protein 2 (HMGA2), a non-histone nuclear-binding protein, is an important regulator of cell growth and differentiation that belongs to the HMGA protein family. ${ }^{15}$ It is an oncofetal protein overexpressed in embryonic tissues and many malignant neoplasms, including lung carcinoma, breast carcinoma, ovarian carcinoma, hepatocellular carcinoma, and malignant gliomas. ${ }^{15-19}$ In several solid cancers, the expression levels of HMGA2 were shown to be positively correlated with tumor progression, metastasis, and poor prognosis. ${ }^{15,20-22}$ However, studies assessing HMGA2 in NPC patients are scarce.

Although HMGA2 has also been found to play a critical role in EMT, inducing epithelial cancer invasion and metastasis, ${ }^{23,24}$ the interaction between expression levels of HMGA2 and EMT markers in NPC remains unclear. Therefore, this study aimed to assess the expression of HMGA2 and EMT-related markers in NPC tissues and analyze the association of HMGA2, E-cadherin, and vimentin with clinicopathological factors and patient OS.

\section{Patients and methods}

\section{Patients and specimens}

Paraffin-embedded biopsies of 124 primary NPC tissues and 20 non-tumoral inflammatory nasopharynx tissues were obtained retrospectively from the Affiliated Jiangsu Cancer Hospital, Nanjing Medical University between May 2006 and May 2011. Inclusion criteria were: 1) no radiotherapy or chemotherapy before biopsy; 2) histopathological diagnosis of NPC; 3) no distant metastasis; and 4) availability of original medical records data and complete follow-up data.

The subjects comprised 90 males and 34 females, with ages ranging from 18-74 years (median: 48 years). According to the TNM classification of Union for International Cancer Control (UICC, 2010), 44 and 80 patients presented with I-II and III-IVa-b disease stages, respectively. Among the 124 cases, 23 had lymph node or distant metastasis by imaging evaluation within 2 years after treatment. The follow-up ended in May 2014, with a median follow-up time of 50.5 months (range: 5-93 months).

This study was approved by the Ethics Committee of Jiangsu Cancer Hospital, the People's Republic of China.

\section{Immunohistochemistry}

To assess the expression of HMGA2, E-cadherin, and vimentin, immunohistochemistry was performed according to the standard streptavidin peroxidase technique. Paraffinembedded tissues were sectioned at $4 \mu \mathrm{m}$ thickness, dewaxed, and rehydrated by routine techniques. All slides were subjected to the heat-induced antigen retrieval method using Tris $(0.01 \mathrm{mmol} / \mathrm{L}, \mathrm{pH}=6.0)$ buffer in a pressure cooker. Then, slides were placed in 3\% hydrogen peroxide for 10 minutes to quench endogenous peroxidase. After rinsing thoroughly with phosphate-buffered saline (PBS) three times for 1 minute each, sections were further incubated with anti-HMGA2 rabbit polyclonal antibody (1:60; Abcam Ltd., Cambridge, UK), anti-E-cadherin mouse monoclonal antibody (1:120; Fuzhou Maixin Biotech, Inc., Fuzhou, People's Republic of China), and anti-vimentin mouse monoclonal antibody (1:100; Fuzhou Maixin Biotech, Inc., Fuzhou, People's Republic of China), respectively, overnight at $4^{\circ} \mathrm{C}$. After washing in PBS, the sections were incubated with biotinylated secondary antibodies (Dako Denmark A/S, Glostrup, Denmark) for 30 minutes at room temperature, and stained with freshly prepared $3,3^{\prime}$ diaminobenzidine and light hematoxylin as counterstain. Known positive controls were included in every staining procedure. PBS was used to replace primary antibodies in the negative control.

\section{Immunohistochemical evaluation}

Tumor and normal tissues were histologically confirmed by hematoxylin and eosin staining. The diagnosis and immunostaining results were confirmed independently by two experienced pathologists who were blinded to the clinical data. In each case, four representative areas were selected and at least 400 tumor cells were observed at $400 \times$ magnification. The percentage of positive cells was evaluated according to the number of positive cells divided by all cancer cells under a microscope for four selected foci. The following proportion scale was adopted: 0 , no positive tumor cells; $1,1 \%-10 \%$ positive tumor cells; $2,11 \%-50 \% ; 3,51 \%-100 \%$. The stating intensity was interpreted by the presence of yellow- or 
brown-colored end product at the target antigen site. Intensity of staining (no staining, mild, moderate, and intense staining) was noted and graded as $0,1,2$, or 3 points, respectively ( 0 , no detectable signal; 1 , mild staining -light yellow color; 2 , moderate - yellow color; 3 , intense - brown color). The final scores were obtained by multiplying the positive tumor grade by the tumor staining intensity score $(0,1,2,3,4,6$, and 9). Final scores $\leq 4$ and $\geq 6$ were regarded as tumors with low and high expression, respectively. ${ }^{13}$

\section{Statistical analysis}

Statistical analysis was performed using SAS software (v9.2; SAS Institute Inc., Cary, NC, USA). The expression levels of HMGA-2, E-cadherin, and vimentin and the relationships between these markers and clinicopathological parameters were analyzed using the chi-square test. The correlations between HMGA-2, E-cadherin, and vimentin were assessed by Spearman's rank test. For survival data, Kaplan-Meier curves were generated, and statistical analysis was carried out using the log-rank test. OS was defined as the time elapsed between the beginning of radiotherapy and death from any cause or the last follow-up date. Cox regression was used for univariate analysis. Variables with prognostic value $(P<0.05)$ in univariate analysis were selected in the final multivariable Cox proportional hazards model, while variables significantly associated with others were excluded from the final multivariable Cox proportional hazards model. $P<0.05$ was considered statistically significant, and all tests were two-sided.

\section{Results}

\section{Protein expression of HMGA2, $\mathrm{E}$-cadherin, and vimentin in NPC and non-tumoral samples}

Immunohistochemical staining of HMGA2, E-cadherin, and vimentin was performed on 124 NPC samples and 20 non-tumoral inflammatory nasopharynx tissues. HMGA2 immunoreactivity was predominantly detected in the nuclei, although a weak cytoplasmic staining was observed. Among the 20 non-tumoral inflammatory nasopharynx tissues, no HMGA2 was detected; of the 124 NPC cases, 54 (43.55\%) exhibited high HMGA2 expression (Figures $1 \mathrm{~A}$ and D), indicating significant differences between the two groups $\left(\chi^{2}=16.121\right.$, $P<0.001)$. Diffuse membrane staining for E-cadherin was detected in cancer cells (Figures 1B and E) and non-tumoral inflammatory nasopharynx tissues. High E-cadherin expression was obtained in 54/124 (43.55\%) and 15/20 (75.00\%) of NPC samples (Figures 1B and E) and non-tumoral inflammatory nasopharynx tissues, respectively $\left(\chi^{2}=6.826, P<0.009\right)$.
Vimentin was mainly localized in the membranes and/or cytoplasm (Figures $1 \mathrm{C}$ and $\mathrm{F}$ ). High vimentin expression was observed in 78/124 (62.90\%) NPC specimens (Figures 1C and $\mathrm{F}$ ), whereas no non-tumoral inflammatory nasopharynx tissues $(0 / 20)$ expressed this protein $\left(\chi^{2}=27.449, P<0.0001\right)$. See supplementary material (Figure S1) for the corresponding HE staining of Figure 1.

\section{Correlation of HMGA2, E-cadherin, and vimentin expression with clinicopathological features}

The clinicopathological characteristics of the patients are summarized in Table 1. Immunohistochemical analyses of NPC tissues showed that HMGA2 protein expression correlated significantly with tumor N stage, TNM stage, and 2-year metastasis status ( $P=0.008,0.026$, and $<0.001$, respectively); meanwhile, no significant correlation was observed with patient sex, age, histologic type, and T stage $(P>0.05)$. Similarly, E-cadherin and vimentin expression levels were closely correlated with $\mathrm{N}$ stage, TNM stage, and 2-year metastasis status (Table 1). To assess the association between the expression levels of HMGA2, E-cadherin, and vimentin, Spearman's correlation analysis was used. Interestingly, high vimentin expression levels were correlated with decreased E-cadherin expression $(r=-0.605, P<0.001)$ in NPC tissues. Furthermore, HMGA2 expression was positively correlated with vimentin levels $(r=0.431, P<0.001)$ and negatively correlated with E-cadherin amounts $(r=-0.413, P<0.001)$ in NPC.

\section{Univariate and multivariate analyses of prognostic factors}

Kaplan-Meier analysis was used to determine the prognostic value of HMGA2 and EMT-related proteins. Of the 124 NPC patients, a cumulative 3-year survival rate of $78.8 \%$ (95\% confidence interval [CI]: 0.708-0.852) was obtained. Interestingly, the cumulative 3-year survival rate was $90.8 \%$ (95\% CI: 0.806-0.957) in the low HMGA2 group, and only $66.1 \%$ (95\% CI: 0.525-0.766) in the high-expression group (Figure 2B). In addition, a cumulative 3-year survival rate of $89.1 \%$ (95\% CI: $0.757-0.953)$ was obtained in the low vimentin group, whereas only $71.8 \%$ (95\% CI: $0.604-0.804)$ of patients survived in the high-expression group (Figure 2C). In the high E-cadherin expression group, a cumulative 3-year survival rate of $88.8 \%$ (95\% CI: $0.768-0.948)$ was obtained, with only $70.0 \%$ (95\% CI: $0.578-0.792)$ in the low-expression group (Figure 2D). A univariate analysis showed significant correlations for T stage $(P=0.01), \mathrm{N}$ stage 

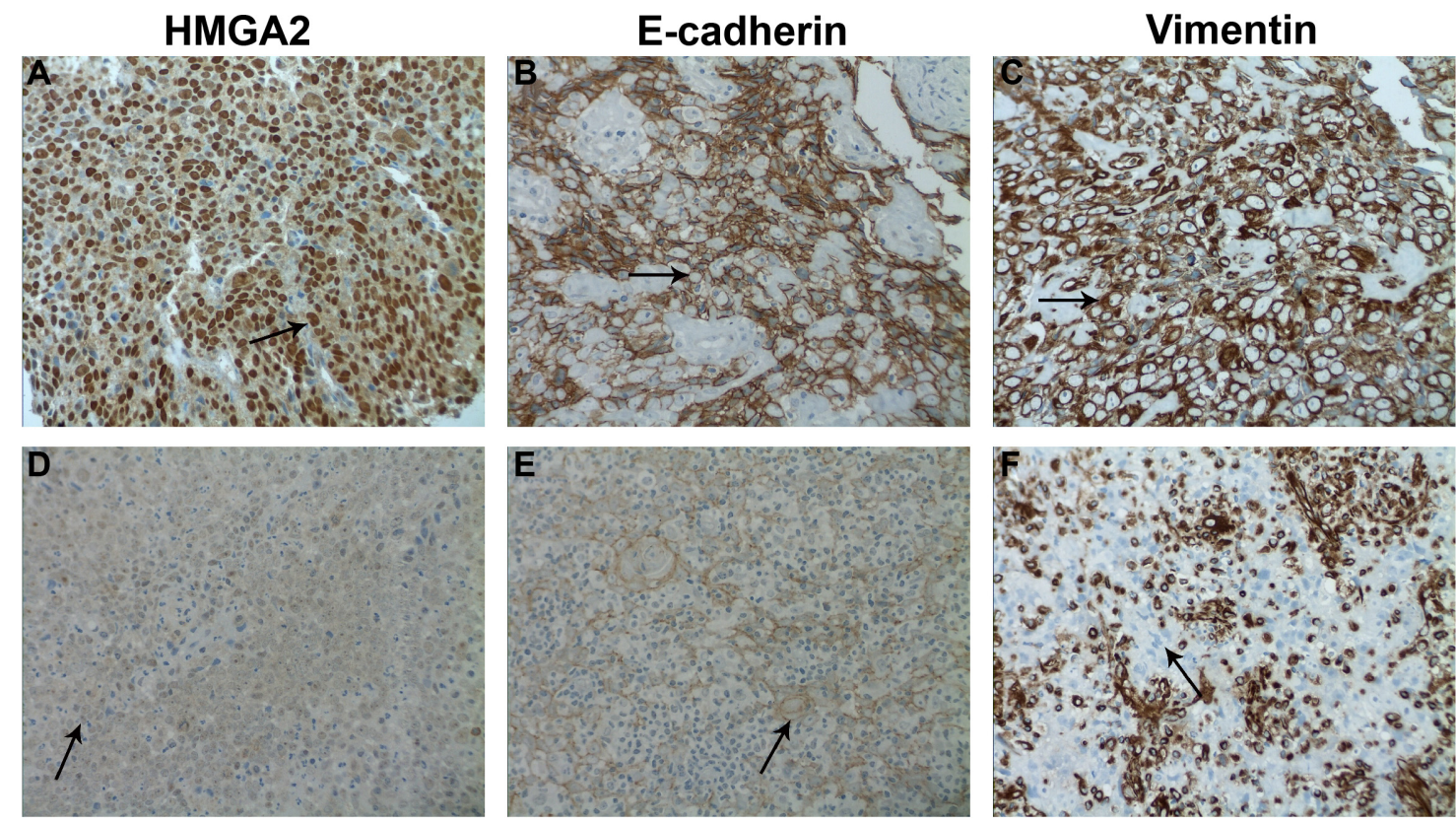

Figure I Immunohistochemistry staining for HMGA2, E-cadherin, and vimentin in human nasopharyngeal carcinoma specimens.

Notes: Representative images show (A) high expression of HMGA2 (brown granules, mainly in the nuclei, $\uparrow$ ), (B) high expression of E-cadherin (brown granules, mainly in the cell membranes, $\uparrow$ ) and (C) high expression of vimentin (brown granules, mainly in the NPC cells membranes and cytoplasm, $\uparrow$ ) in tumor cells. Representative images show (D) low expression of HMGA2 (light yellow granules, mainly in the nuclei, $\uparrow$ ), (E) low expression of E-cadherin (light yellow granules, mainly in the cell membranes, $\uparrow$ ) and (F) low expression of vimentin (no staining in the NPC cells, $\uparrow$ ), and high expression of vimentin in mesenchymal cells. All images, 400x.

Abbreviations: HMGA2, high-mobility group protein 2; NPC, nasopharyngeal carcinoma.

Table I Relationship between clinicopathological features and immunostaining results

\begin{tabular}{|c|c|c|c|c|c|c|c|c|c|c|}
\hline \multirow{2}{*}{$\begin{array}{l}\text { Clinical } \\
\text { parameter }\end{array}$} & \multirow[t]{2}{*}{$\mathbf{n}$} & \multicolumn{3}{|c|}{ HMGA2 } & \multicolumn{3}{|c|}{ Vimentin } & \multicolumn{3}{|c|}{ E-cadherin } \\
\hline & & High & Low & $P$-value & High & Low & $P$-value & High & Low & $P$-value \\
\hline \multicolumn{11}{|l|}{ Sex } \\
\hline Male & 90 & 40 & 50 & & 57 & 33 & & 37 & 53 & \\
\hline Female & 34 & 19 & 15 & 0.255 & 21 & 13 & 0.872 & 17 & 17 & 0.373 \\
\hline \multicolumn{11}{|l|}{ Age (years) } \\
\hline$<50$ & 69 & 30 & 39 & & 46 & 23 & & 28 & 41 & \\
\hline$\geq 50$ & 55 & 29 & 26 & 0.306 & 32 & 23 & 0.331 & 26 & 29 & 0.455 \\
\hline \multicolumn{11}{|c|}{ Histologic type } \\
\hline NKC & 11 & 4 & 7 & & 8 & 3 & & 5 & 6 & \\
\hline $\mathrm{KSCC}$ & 113 & 63 & 50 & 0.218 & 70 & 43 & 0.480 & 39 & 74 & 0.469 \\
\hline \multicolumn{11}{|l|}{ T stage } \\
\hline TI-2 & 68 & 28 & 40 & & 40 & 28 & & 31 & 37 & \\
\hline T3-4 & 56 & 31 & 25 & 0.116 & 38 & 18 & 0.300 & 23 & 33 & 0.614 \\
\hline \multicolumn{11}{|l|}{$N$ stage } \\
\hline No-I & 66 & 24 & 42 & & 35 & 31 & & 35 & 31 & \\
\hline N2-3 & 58 & 35 & 23 & 0.008 & 43 & 15 & 0.015 & 19 & 39 & 0.023 \\
\hline \multicolumn{11}{|l|}{ TNM stage } \\
\hline I-II & 44 & 15 & 29 & & 20 & 24 & & 27 & 17 & \\
\hline III-IV & 80 & 44 & 36 & 0.026 & 58 & 22 & 0.003 & 27 & 53 & 0.003 \\
\hline \multicolumn{11}{|l|}{ Metastasis* } \\
\hline No & 101 & 38 & 63 & & 56 & 45 & & 49 & 52 & \\
\hline Yes & 23 & 21 & 2 & 0.0001 & 22 & I & 0.0003 & 5 & 18 & 0.019 \\
\hline
\end{tabular}

Note: *Metastasis: within 2 years after treatment.

Abbreviations: HMGA2, high-mobility group protein 2; NKC, non-keratinizing carcinoma; KSCC, keratinizing squamous cell carcinoma. 
A

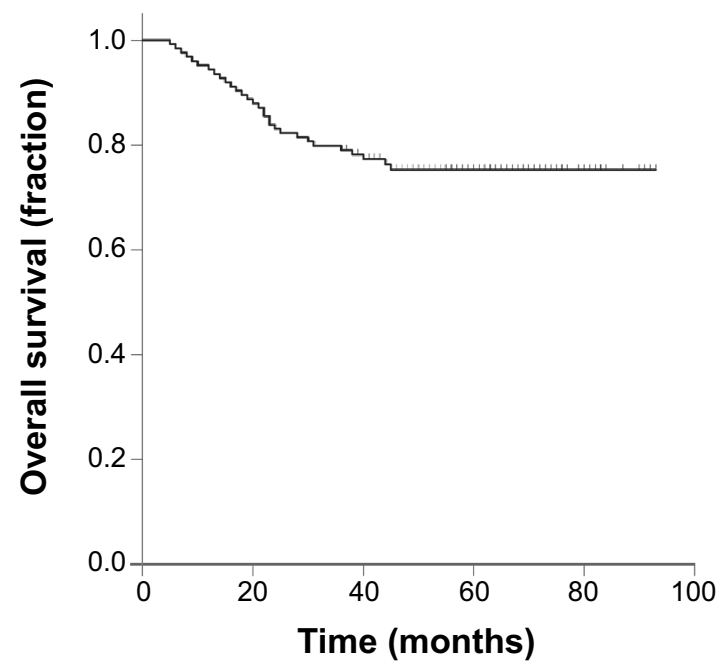

C

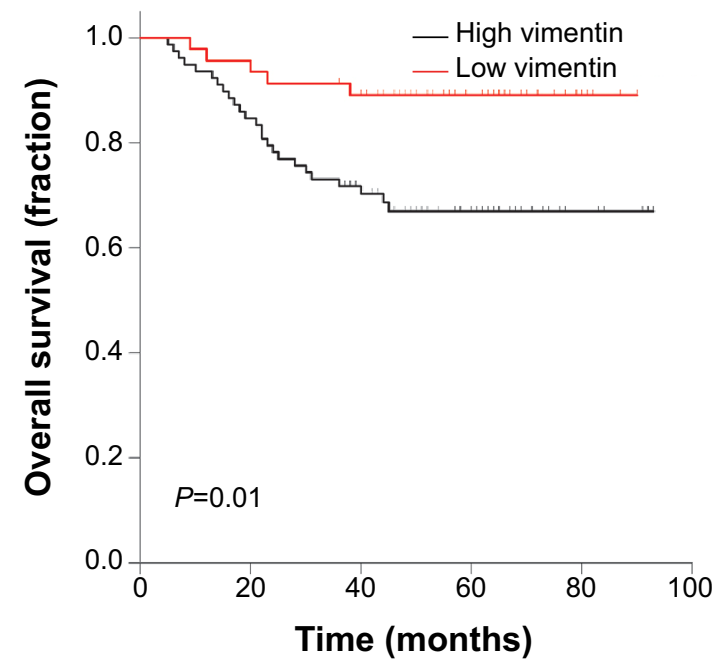

B

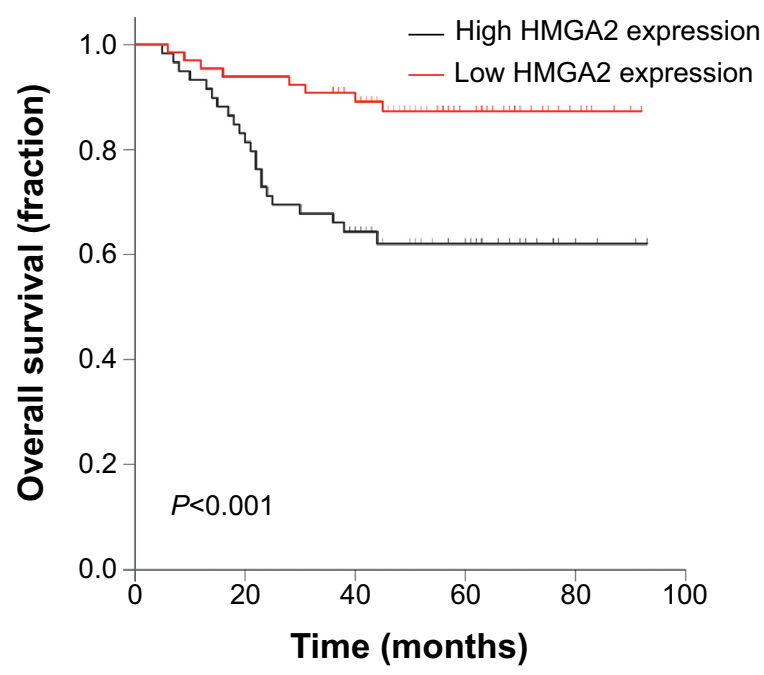

D

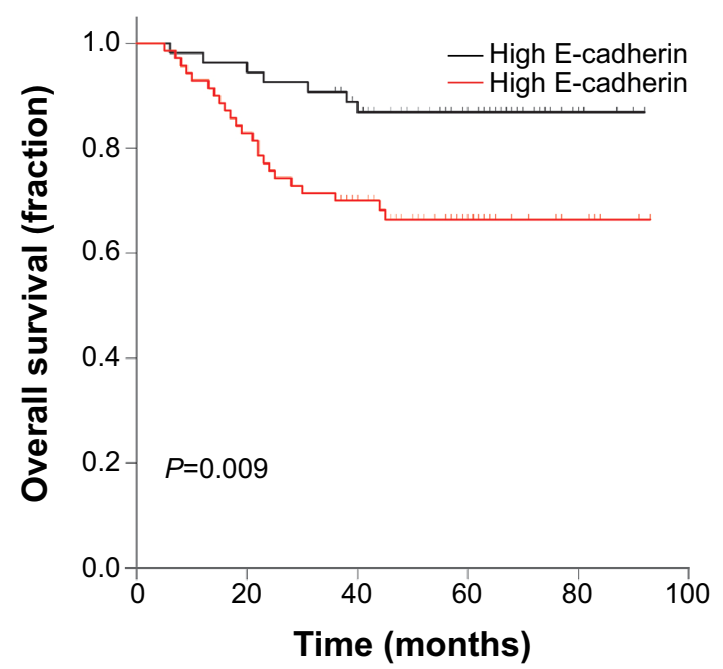

Figure 2 (A) Kaplan-Meier survival curves of overall survival in 124 patients with NPC and overall survival curves based on (B) HMGA2, (C) vimentin, and (D) E-cadherin expression levels.

Note: $P$-values were calculated by the log-rank test.

Abbreviations: HMGA2, high-mobility group protein 2; NPC, nasopharyngeal carcinoma.

$(P<0.001)$, TNM stage $(P=0.003)$, HMGA2 $(P=0.002)$, E-cadherin $(P=0.014)$, and vimentin $(P=0.013)$ with a poor survival prognosis. In multivariate analysis of significant factors, only $\mathrm{N}$ stage (hazard ratio [HR]: 7.892, 95\% CI: 2.731-22.807, $P<0.001$ ) and HMGA-2 (HR: 2.683, 95\% CI: $1.185-6.077, P=0.018)$ were independently associated with OS (Table 2).

\section{Discussion}

In the present study, we demonstrated that HMGA2 and EMTrelated markers are differentially expressed between NPC and non-tumoral tissues. Additionally, we found in NPC tissues, for the first time, a significant association between protein expression of HMGA2 and EMT indicators, and these proteins were closely linked with cancer progression and metastasis. Moreover, our results indicated that HMGA2 expression is an independent prognostic indicator for OS in NPC.

Previous studies have revealed that HMGA2 is expressed mainly in the early embryo and suppressed in human adult tissues. In addition, elevated expression of HMGA2 has been observed in a variety of cancers. ${ }^{23,25}$ In agreement with these reports, we found that HMGA2 expression is higher in NPC compared with non-tumorous tissues. The differential expression of HMGA2 and EMT-related proteins between non-tumorous and NPC tissues suggest a potential role for HMGA2 and EMT-related proteins in NPC carcinogenesis. 
Table 2 Univariate and multivariate Cox regression analysis of overall survival in NPC patients

\begin{tabular}{|c|c|c|c|c|c|c|}
\hline \multirow[t]{2}{*}{ Parameters } & \multicolumn{3}{|c|}{ Univariate analysis } & \multicolumn{3}{|c|}{ Multivariate analysis } \\
\hline & HR & $95 \% \mathrm{Cl}$ & $P$-value & HR & $95 \% \mathrm{Cl}$ & $P$-value \\
\hline \multicolumn{7}{|l|}{ Sex } \\
\hline Male vs female & 1.161 & $0.532-2.536$ & 0.707 & - & - & - \\
\hline \multicolumn{7}{|l|}{ Age (years) } \\
\hline$<50$ vs $\geq 50$ & 0.989 & $0.961-1.017$ & 0.427 & - & - & - \\
\hline \multicolumn{7}{|l|}{ Histologic type } \\
\hline NKC vs KSCC & 1.642 & $0.482-2.439$ & $0.64 I$ & - & - & - \\
\hline \multicolumn{7}{|l|}{ T stage } \\
\hline TI -2 vs T3-4 & 2.708 & I.267-5.786 & 0.010 & - & - & - \\
\hline \multicolumn{7}{|l|}{$\mathrm{N}$ stage } \\
\hline N0-I vs N2-3 & 9.260 & $3.228-26.563$ & $<0.001$ & 7.892 & $2.73 \mathrm{I}-22.807$ & $<0.001$ \\
\hline \multicolumn{7}{|l|}{ TNM stage } \\
\hline I-II vs III-IV & 9.086 & $2.164-38.153$ & 0.003 & - & - & - \\
\hline \multicolumn{7}{|l|}{ HMGA2 } \\
\hline Low vs high & 3.596 & $1.598-8.092$ & 0.002 & 2.683 & I.I85-6.077 & 0.018 \\
\hline \multicolumn{7}{|l|}{ Vimentin } \\
\hline Low vs high & 3.364 & I.287-8.793 & 0.013 & - & - & - \\
\hline \multicolumn{7}{|l|}{ E-cadherin } \\
\hline Low vs high & 0.344 & $0.147-0.802$ & 0.014 & - & - & - \\
\hline
\end{tabular}

Abbreviations: $\mathrm{Cl}$, confidence interval; HMGA2, high-mobility group protein 2; HR, hazard ratio; NPC, nasopharyngeal carcinoma; NKC, non-keratinizing carcinoma; KSCC, keratinizing squamous cell carcinoma.

These findings are consistent with previous studies described in other carcinomas and non-tumorous tissues or tumor cell lines. ${ }^{26,27}$

It is generally accepted that reduced E-cadherin expression coupled with increased vimentin expression is characteristic of EMT. ${ }^{7,28}$ Recent studies have demonstrated that ectopic expression of HMGA2 in epithelial cells induces EMT, which has been implicated in the acquisition of metastatic properties in tumor cells. ${ }^{29-33}$ For instance, Zha et al showed that in gastric cancer, HMGA2 overexpression induces protein changes consistent with EMT and enhanced epithelial cell invasion and migration both in vitro and in vivo. ${ }^{30}$ Similarly, Wu et al found that HMGA2 silencing in ovarian cancer cell lines partially suppressed the aggressiveness of tumor cells, and caused changes in the expression of several EMT-associated genes, including vimentin and E-cadherin. ${ }^{31}$ In NPC specimens, for the first time, the present results demonstrated that high HMGA2 expression is significantly and positively associated with vimentin amounts and negatively with E-cadherin levels. This finding corroborated with previous evidence supporting the potential role of HMGA2 in EMT, which is associated with vimentin expression and E-cadherin absence in NPC patients. In addition, the previous study has demonstrated that expressions of HMGA2, E-cadherin, and vimentin are correlated with $\mathrm{T}$ stage and bladder cancer recurrence. ${ }^{29} \mathrm{~A}$ relevant finding in the present study is that HMGA2, E-cadherin, and vimentin expression levels were all significantly correlated with various aggressive behaviors, including N stage, TNM stage, and 2-year metastasis status in NPC. This finding suggests that the biomarkers may have important roles in the development and metastasis of NPC. However, no association was found with $\mathrm{T}$ stage. These discrepancies between the present study and the previous study might have resulted from the different antibodies used, varying staining evaluation methods, and the different biological functions of HMGA2 in various carcinomas.

Finally, a significant association between HMGA2 expression and poor prognosis has been reported in various tumor types. ${ }^{34,35}$ In agreement with these findings, our results provide evidence that HMGA2 might be considered a valuable prognostic biomarker in NPC. Currently, high expression of cytoplasmic E-cadherin and nuclear vimentin are considered independent prognostic factors for poor survival of NPC patients. ${ }^{13}$ However, we only found HMGA2 expression and $\mathrm{N}$ stage as independent predictors of poor prognosis by multivariate analyses. These variations may be due to the different study populations evaluated and various clinical data quality. Additionally, another report suggested that the molecular mechanism of HMGA2 possibly involves the activation of the TGF $\beta$ signaling pathway in epithelial carcinomas. ${ }^{23}$ In addition, it was shown that the genes making up the HMGA2-TET1-HOXA9 pathway are coordinately regulated in breast cancer, and together encompass a prognostic signature for patient survival. ${ }^{36}$ Future studies should determine the molecular mechanism by which HMGA2 induces progression and metastasis in NPC cell lines. 
In summary, our results suggested that HMGA2 and EMT protein expression levels are associated with progression and metastasis of NPC. HMGA2 may promote metastasis through EMT in NPC patients and be used as a prognostic indicator. These protein markers may have significant effects on targeted therapy; however, larger prospective studies are required to further validate these findings.

\section{Acknowledgments}

The authors thank the Department of Pathology, The Affiliated Jiangsu Cancer Hospital of Nanjing Medical University for kindly providing paraffin-embedded biopsies and the members of the Research Center of Clinical Oncology, The Affiliated Jiangsu Cancer Hospital of Nanjing Medical University for their technical assistance.

\section{Disclosure}

The authors report no conflicts of interest in this work.

\section{References}

1. Jemal A, Bray F, Center MM, Ferlay J, Ward E, Forman D. Global cancer statistics. CA Cancer J Clin. 2011;61(2):69-90.

2. Wei WI, Sham JS. Nasopharyngeal carcinoma. Lancet. 2005;365(9476); 2041-2054.

3. Huang T, Chen MH, Wu MY, Wu XY. Correlation between expression of extracellular matrix metalloproteinase inducer and matrix metalloproteinase-2 and cervical lymph node metastasis of nasopharyngeal carcinoma. Ann Otol Rhinol Laryngol. 2013;122(3):210-215.

4. Huang CJ, Leung SW, Lian SL, Wang CJ, Fang FM, Ho YH. Patterns of distant metastases in nasopharyngeal carcinoma. Kaohsiung $J$ Med Sci. 1996;12(4):229-234.

5. Erkal HS, Serin M, Cakmak A. Nasopharyngeal carcinomas: analysis of patient, tumor and treatment characteristics determining outcome. Radiother Oncol. 2001;61(3):247-256.

6. Gao D, Vahdat LT, Wong S, Chang JC, Mittal V. Microenvironmental regulation of epithelial-mesenchymal transitions in cancer. Cancer Res. 2012;72(19):4883-4889.

7. Lim J, Thiery JP. Epithelial-mesenchymal transitions: insights from development. Development. 2012;139(19):3471-3486.

8. Todosi AM, Gavrilescu MM, Anitei GM, Filip B, Scripcariu V. Colon cancer at the molecular level - usefulness of epithelial-mesenchymal transition analysis. Rev Med Chir Soc Med Nat Iasi. 2012;116(4):1106-1111.

9. Smith A, Teknos TN, Pan Q. Epithelial to mesenchymal transition in head and neck squamous cell carcinoma. Oral Oncol. 2013;49(4):287-292.

10. Liu AN, Zhu ZH, Chang SJ, Hang XS. Twist expression associated with the epithelial-mesenchymal transition in gastric cancer. Mol Cell Biochem. 2012;367(1-2):195-203.

11. Tian W, Wang G, Yang J, Pan Y, Ma Y. Prognostic role of E-cadherin and Vimentin expression in various subtypes of soft tissue leiomyosarcomas. Med Oncol. 2013;30(1):401.

12. Kong FF, Qu ZQ, Yuan HH, et al. Overexpression of FOXM1 is associated with EMT and is a predictor of poor prognosis in non-small cell lung cancer. Oncol Rep. 2014;31(6):2660-2668.

13. Luo W, Fang W, Li S, Yao K. Aberrant expression of nuclear vimentin and related epithelial-mesenchymal transition markers in nasopharyngeal carcinoma. Int J Cancer. 2012;131(8):1863-1873.

14. Stoyianni A, Goussia A, Pentheroudakis G, et al. Immunohistochemical study of the epithelial-mesenchymal transition phenotype in cancer of unknown primary: incidence, correlations and prognostic utility. Anticancer Res. 2012;32(4):1273-1281.
15. Kumar MS, Armenteros-Monterroso E, East $\mathrm{P}$, et al. HMGA2 functions as a competing endogenous RNA to promote lung cancer progression. Nature. 2014;505(7482):212-217.

16. Sun M, Gomes S, Chen P, et al. RKIP and HMGA2 regulate breast tumor survival and metastasis through lysyl oxidase and syndecan-2. Oncogene. 2014;33(27):3528-3537.

17. Wu J, Wei JJ. HMGA2 and high-grade serous ovarian carcinoma. $J$ Mol Med (Berl). 2013;91(10):1155-1165.

18. Luo Y, Li W, Liao H. HMGA2 induces epithelial-to-mesenchymal transition in human hepatocellular carcinoma cells. Oncol Lett. 2013;5(4): $1353-1356$.

19. Liu B, Pang B, Hou X, et al. Expression of high-mobility group AThook protein 2 and its prognostic significance in malignant gliomas. Hum Pathol. 2014;45(8):1752-1758.

20. Di Cello F, Hillion J, Hristov A, et al. HMGA2 participates in transformation in human lung cancer. Mol Cancer Res. 2008;6(5):743-750.

21. Mahajan A, Liu Z, Gellert L, et al. HMGA2: a biomarker significantly overexpressed in high-grade ovarian serous carcinoma. Mod Pathol. 2010; 23(5):673-681.

22. Yang GL, Zhang LH, Bo JJ, et al. Overexpression of HMGA2 in bladder cancer and its association with clinicopathologic features and prognosis HMGA2 as a prognostic marker of bladder cancer. Eur J Surg Oncol. 2011;37(3):265-271.

23. Morishita A, Zaidi MR, Mitoro A, et al. HMGA2 is a driver of tumor metastasis. Cancer Res. 2013;73(14):4289-4299.

24. Thuault S, Tan EJ, Peinado H, Cano A, Heldin $\mathrm{CH}$, Moustakas A. HMGA2 and Smads co-regulate SNAIL1 expression during induction of epithelial-to-mesenchymal transition. J Biol Chem. 2008;283(48):33437-33446.

25. Malek A, Bakhidze E, Noske A, et al. HMGA2 gene is a promising target for ovarian cancer silencing therapy. Int J Cancer. 2008;123(2):348-356.

26. Baumgart E, Cohen MS, Silva Neto B, et al. Identification and prognostic significance of an epithelial-mesenchymal transition expression profile in human bladder tumors. Clin Cancer Res. 2007;13(6):1685-1694.

27. Liu LK, Jiang XY, Zhou XX, Wang DM, Song XL, Jiang HB. Upregulation of vimentin and aberrant expression of E-cadherin/betacatenin complex in oral squamous cell carcinomas: correlation with the clinicopathological features and patient outcome. Mod Pathol. 2010;23(2):213-224.

28. Lee WY, Shin DY, Kim HJ, Ko YH, Kim S, Jeong HS. Prognostic significance of epithelial-mesenchymal transition of extracapsular spread tumors in lymph node metastases of head and neck cancer. Ann Surg Oncol. 2014;21(6):1904-1911.

29. Ding X, Wang Y, Ma X, et al. Expression of HMGA2 in bladder cancer and its association with epithelial-to-mesenchymal transition. Cell Prolif. 2014;47(2):146-151.

30. Zha L, Zhang J, Tang W, et al. HMGA2 elicits EMT by activating the Wnt/beta-catenin pathway in gastric cancer. Dig Dis Sci. 2013;58(3): 724-733.

31. Wu J, Liu Z, Shao C, et al. HMGA2 overexpression-induced ovarian surface epithelial transformation is mediated through regulation of EMT genes. Cancer Res. 2011;71(2):349-359.

32. Zhu C, Li J, Cheng G, et al. miR-154 inhibits EMT by targeting HMGA2 in prostate cancer cells. Mol Cell Biochem. 2013;379(1-2):69-75.

33. Watanabe S, Ueda Y, Akaboshi S, Hino Y, Sekita Y, Nakao M. HMGA2 maintains oncogenic RAS-induced epithelial-mesenchymal transition in human pancreatic cancer cells. Am J Pathol. 2009;174(3):854-868.

34. Yamazaki H, Mori T, Yazawa M, et al. Stem cell self-renewal factors Bmi1 and HMGA2 in head and neck squamous cell carcinoma: clues for diagnosis. Lab Invest. 2013;93(12):1331-1338.

35. Raskin L, Fullen DR, Giordano TJ, et al. Transcriptome profiling identifies HMGA2 as a biomarker of melanoma progression and prognosis. J Invest Dermatol. 2013;133(11):2585-2592.

36. Sun M, Song CX, Huang H, et al. HMGA2/TET1/HOXA9 signaling pathway regulates breast cancer growth and metastasis. Proc Natl Acad Sci U S A. 2013;110(24):9920-9925. 


\section{Supplementary material}
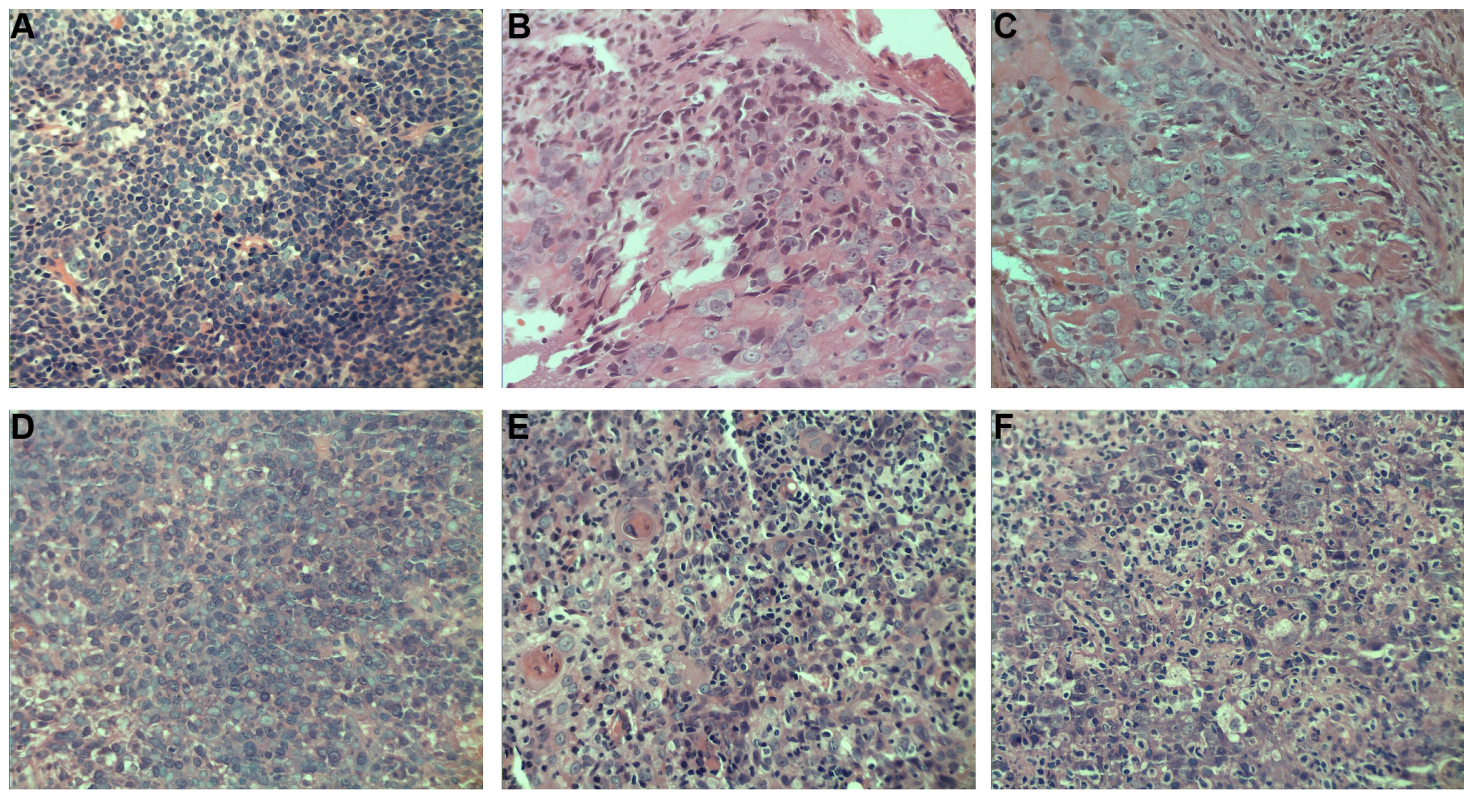

Figure SI Nasopharyngeal carcinoma specimens by HE staining.

Notes: Representative HE staining images for samples in (A) nasopharyngeal squamous cell carcinoma (high expression of HMGA2), (B) nasopharyngeal squamous cell carcinoma (high expression of E-cadherin), (C) nasopharyngeal squamous cell carcinoma (high expression of vimentin), (D) nasopharyngeal squamous cell carcinoma (low expression of HMGA2), (E) nasopharyngeal squamous cell carcinoma (low expression of E-cadherin), (F) nasopharyngeal squamous cell carcinoma (low expression of vimentin). All images, 400X.

Abbreviations: HMGA2, high-mobility group protein 2; HE, hematoxylin and eosin.

\section{Publish your work in this journal}

OncoTargets and Therapy is an international, peer-reviewed, open access journal focusing on the pathological basis of all cancers, potential targets for therapy and treatment protocols employed to improve the management of cancer patients. The journal also focuses on the impact of management programs and new therapeutic agents and protocols on patient perspectives such as quality of life, adherence and satisfaction. The manuscript management system is completely online and includes a very quick and fair peer-review system, which is all easy to use. Visit http://www.dovepress.com/testimonials.php to read real quotes from published authors. 